\title{
ON SHATTERING, SPLITTING AND REAPING PARTITIONS
}

\author{
Lorenz Halbeisen円 \\ Université de Caen
}

France

Keywords: Cardinal invariants, partition properties, dual-Mathias forcing.

MS-Classification: 03E05, 03E35, 03C25, 04A20, 05A18.

\begin{abstract}
In this article we investigate the dual-shattering cardinal $\mathfrak{H}$, the dualsplitting cardinal $\mathfrak{S}$ and the dual-reaping cardinal $\mathfrak{R}$, which are dualizations of the well-known cardinals $\mathfrak{h}$ (the shattering cardinal, also known as the distributivity number of $\mathcal{P}(\omega) / f i n), \mathfrak{s}$ (the splitting number) and $\mathfrak{r}$ (the reaping number). Using some properties of the ideal $\mathfrak{J}$ of nowhere dual-Ramsey sets, which is an ideal over the set of partitions of $\omega$, we show that $\operatorname{add}(\mathfrak{J})=\operatorname{cov}(\mathfrak{J})=\mathfrak{H}$. With this result we can show that $\mathfrak{H}>\omega_{1}$ is consistent with ZFC and as a corollary we get the relative consistency of $\mathfrak{H}>\mathfrak{t}$, where $\mathfrak{t}$ is the tower number. Concerning $\mathfrak{S}$ we show that $\operatorname{cov}(\mathcal{M}) \leq \mathfrak{S}$ (where $\mathcal{M}$ is the ideal of the meager sets). For the dualreaping cardinal $\mathfrak{R}$ we get $\mathfrak{p} \leq \mathfrak{R} \leq \mathfrak{r}$ (where $\mathfrak{p}$ is the pseudo-intersection number) and for a modified dual-reaping number $\mathfrak{R}^{\prime}$ we get $\mathfrak{R}^{\prime} \leq \mathfrak{d}$ (where $\mathfrak{d}$ is the dominating number). As a consistency result we get $\mathfrak{R}<\operatorname{cov}(\mathcal{M})$.
\end{abstract}

\section{The set of partitions}

A partial partition $X$ (of $\omega$ ) consisting of pairwise disjoint, nonempty sets, such that $\operatorname{dom}(X):=\bigcup X \subseteq \omega$. The elements of a partial partition $X$ are called the blocks of $X$ and $\operatorname{Min}(X)$ denotes the set of the least elements of the blocks of $X$. If $\operatorname{dom}(X)=\omega$, then $X$ is called a partition. $\{\omega\}$ is the partition such that each block is a singleton and $\{\{\omega\}\}$ is the partition containing only one block. The set of all partitions containing infinitely (resp. finitely) many blocks is denoted by $(\omega)^{\omega}$ (resp. $\left.(\omega)^{<\omega}\right)$. By $(\omega)^{\underline{\omega}}$ we denote the set of all infinite partitions such that at least one block is infinite. The set of all partial partitions with $\operatorname{dom}(X) \in \omega$ is denoted by $(\mathbb{N})$.

Let $X_{1}, X_{2}$ be two partial partitions. We say that $X_{1}$ is coarser than $X_{2}$, or that $X_{2}$ is finer than $X_{1}$, and write $X_{1} \sqsubseteq X_{2}$ if for all blocks $b \in X_{1}$ the set $b \cap \operatorname{dom}\left(X_{2}\right)$ is the union of some sets $b_{i} \cap \operatorname{dom}\left(X_{1}\right)$, where each $b_{i}$ is a block of $X_{2}$. (Note that if $X_{1}$ is coarser than $X_{2}$, then $X_{1}$ is in a natural way also contained in $X_{2}$.) Let $X_{1} \sqcap X_{2}$ denotes the finest partial partition which is coarser than $X_{1}$ and $X_{2}$ such that $\operatorname{dom}\left(X_{1} \sqcap X_{2}\right)=\operatorname{dom}\left(X_{1}\right) \cup \operatorname{dom}\left(X_{2}\right)$. Similarly $X_{1} \sqcup X_{2}$ denotes the coarsest partial partition which is finer than $X_{1}$ and $X_{2}$ such that $\operatorname{dom}\left(X_{1} \sqcup X_{2}\right)=\operatorname{dom}\left(X_{1}\right) \cup \operatorname{dom}\left(X_{2}\right)$.

\footnotetext{
${ }^{1}$ The author wishes to thank the Swiss National Science Foundation for supporting him.
} 
If $f$ is a finite subset of $\omega$, then $\{f\}$ is a partial partition with $\operatorname{dom}(\{f\})=f$. For two partial partitions $X_{1}$ and $X_{2}$ we write $X_{1} \sqsubseteq^{*} X_{2}$ if there is a finite set $f \subseteq \operatorname{dom}\left(X_{1}\right)$ such that $X_{1} \sqcap\{f\} \sqsubseteq X_{2}$ and say that $X_{1}$ is coarser* than $X_{2}$. If $X_{1} \sqsubseteq^{*} X_{2}$ and $X_{2} \sqsubseteq^{*} X_{1}$ then we write $X_{1} \stackrel{*}{=} X_{2}$. If $X \stackrel{*}{=}\{\omega\}$, then $X$ is called trivial.

Let $X_{1}, X_{2}$ be two partial partitions. If each block of $X_{1}$ can be written as the intersection of a block of $X_{2}$ with $\operatorname{dom}\left(X_{1}\right)$, then we write $X_{1} \preceq X_{2}$. Note that $X_{1} \preceq X_{2}$ implies $\operatorname{dom}\left(X_{1}\right) \subseteq \operatorname{dom}\left(X_{2}\right)$.

We define a topology on the set of partitions as follows. Let $X \in(\omega)^{\omega}$ and $s \in(\mathbb{N})$ such that $s \sqsubseteq X$, then $(s, X)^{\omega}:=\left\{Y \in(\omega)^{\omega}: s \preceq Y \wedge Y \sqsubseteq X\right\}$ and $(X)^{\omega}:=(\emptyset, X)^{\omega}$. Now let the basic open sets on $(\omega)^{\omega}$ be the sets $(s, X)^{\omega}$ (where $X$ and $s$ as above). These sets are called the dual Ellentuck neighborhoods. The topology induced by the dual Ellentuck neighborhoods is called the dual Ellentuck topology (cf. CSS).

\section{On the dual-shattering cardinal $\mathfrak{H}$}

\section{Four cardinals}

We first give the definition of the dual-shattering cardinal $\mathfrak{H}$.

Two partitions $X_{1}, X_{2} \in(\omega)^{\omega}$ are called almost orthogonal $\left(X_{1} \perp_{*} X_{2}\right)$ if $X_{1} \sqcap$ $X_{2} \notin(\omega)^{\omega}$, otherwise they are compatible $\left(X_{1} \| X_{2}\right)$. If $X_{1} \sqcap X_{2}=\{\{\omega\}\}$, then they are called orthogonal $\left(X_{1} \perp X_{2}\right)$. We say that a family $\mathcal{A} \subseteq(\omega)^{\omega}$ is maximal almost orthogonal (mao) if $\mathcal{A}$ is a maximal family of pairwise almost orthogonal partitions. A family $\mathcal{H}$ of mao families of partitions shatters a partition $X \in(\omega)^{\omega}$, if there are $H \in \mathcal{H}$ and two distinct partitions in $H$ which are both compatible with $X$. A family of mao families of partitions is shattering if it shatters each member of $(\omega)^{\omega}$. The dual-shattering cardinal $\mathfrak{H}$ is the least cardinal number $\kappa$, for which there exists a shattering family of cardinality $\kappa$.

One can show that $\mathfrak{H} \leq \mathfrak{h}$ and $\mathfrak{H} \leq \mathfrak{S}$ (cf. CMW]), (where $\mathfrak{S}$ is the dual-splitting cardinal).

\section{Two cardinals related to the ideal of nowhere dual-Ramsey sets}

Let $C \subseteq(\omega)^{\omega}$ be a set of partitions, then we say that $C$ has the dual-Ramsey property or that $C$ is dual-Ramsey, if there is a partition $X \in(\omega)^{\omega}$ such that $(X)^{\omega} \subseteq C$ or $(X)^{\omega} \cap C=\emptyset$. If the latter case holds, we also say that $C$ is dual-Ramseyo. If for each dual Ellentuck neighborhood $(s, Y)^{\omega}$ there is an $X \in(s, Y)^{\omega}$ such that $(s, X)^{\omega} \subseteq C$ or $(s, X)^{\omega} \cap C=\emptyset$, we call $C$ completely dual-Ramsey. If for each dual Ellentuck neighborhood the latter case holds, we say that $C$ is nowhere dual-Ramsey.

REMARK 1: In CS it is proved, that a set is completely dual-Ramsey if and only if it has the Baire property and it is nowhere dual-Ramsey if and only if it is meager with respect to the dual Ellentuck topology. From this it follows, that a set is nowhere dual-Ramsey if and only if the complement contains a dense and open subset (with respect to the dual Ellentuck topology). 
Let $\mathfrak{J}$ be set of partitions which are completely dual-Ramsey。. The set $\mathfrak{J} \subseteq$ $\mathcal{P}\left((\omega)^{\omega}\right)$ is an ideal which is not prime. The cardinals $\operatorname{add}(\mathfrak{J})$ and $\operatorname{cov}(\mathfrak{J})$ are two cardinals related to this ideal.

$\operatorname{add}(\mathfrak{J})$ is the smallest cardinal $\kappa$ such that there exists a family $\mathcal{F}=\left\{J_{\alpha} \in \mathfrak{J}\right.$ : $\alpha<\kappa\}$ with $\bigcup \mathcal{F} \notin \mathfrak{J}$.

$\operatorname{cov}(\mathfrak{J})$ is the smallest cardinal $\kappa$ such that there exists a family $\mathcal{F}=\left\{J_{\alpha} \in \mathfrak{J}\right.$ : $\alpha<\kappa\}$ with $\bigcup \mathcal{F}=(\omega)^{\omega}$.

Because $(\omega)^{\omega} \notin \mathfrak{J}$, it is clear that $\operatorname{add}(\mathfrak{J}) \leq \operatorname{cov}(\mathfrak{J})$. Further it is easy to see that $\omega_{1} \leq \operatorname{add}(\mathfrak{J})$. In the next section we will show that $\operatorname{add}(\mathfrak{J})=\operatorname{cov}(\mathfrak{J})$.

\section{The distributivity number $\mathrm{d}(\mathfrak{W})$}

A complete Boolean algebra $\langle B, \leq\rangle$ is called $\kappa$-distributive, where $\kappa$ is a cardinal, if and only if for every family $\left\langle u_{\alpha i}: i \in I_{\alpha}, \alpha<\kappa\right\rangle$ of members of $B$ the following holds:

$$
\prod_{\alpha<\kappa} \sum_{i \in I_{\alpha}} u_{\alpha i}=\sum_{f \in \prod_{\alpha<\kappa} I_{\alpha}} \prod_{\alpha<\kappa} u_{\alpha f(\alpha)} .
$$

It is well known (cf. [Je2]) that for a forcing notion $\langle P, \leq\rangle$ the following statements are equivalent:

- r.o. $(P)$ is $\kappa$-distributive.

- The intersection of $\kappa$ open dense sets in $P$ is dense.

- Every family of $\kappa$ maximal anti-chains of $P$ has a common refinement.

- Forcing with $P$ does not add a new subset of $\kappa$.

Let $\mathcal{J}$ be the ideal of all finite sets of $\omega$ and let $\left\langle(\omega)^{\omega} / \mathcal{J}, \leq\right\rangle=: \mathfrak{W}$ be the partial order defined as follows:

$$
\begin{gathered}
p \in \mathfrak{W} \Leftrightarrow p \in(\omega)^{\omega}, \\
p \leq q \Leftrightarrow p \sqsubseteq^{*} q .
\end{gathered}
$$

The distributivity number $\mathbf{d}(\mathfrak{W})$ is defined as the least cardinal $\kappa$ for which the Boolean algebra r.o.( $(\mathfrak{W})$ is not $\kappa$-distributive.

\section{The four cardinals are equal}

Now we will show, that the four cardinals defined above are all equal. This is a similar result as in the case when we consider infinite subsets of $\omega$ instead of infinite partitions (cf. [P] and [BPS).

FACT 2.1 If $T \subseteq(\omega)^{\omega}$ is an open and dense set with respect to the dual Ellentuck topology, then it contains a mao family.

PROOF: First choose an almost orthogonal family $\mathcal{A} \subseteq T$ which is maximal in $T$. Now for an arbitrary $X \in(\omega)^{\omega}, T \cap(X)^{\omega} \neq \emptyset$. So, $X$ must be compatible with some $A \in \mathcal{A}$ and therefore $\mathcal{A}$ is mao. $\dashv$ 
LEMMA $2.2 \mathfrak{H} \leq \operatorname{add}(\mathfrak{J})$

ProOF: Let $\left\langle S_{\alpha}: \alpha<\lambda<\mathfrak{H}\right\rangle$ be a sequence of nowhere dual-Ramsey sets and let $T_{\alpha} \subseteq(\omega)^{\omega} \backslash S_{\alpha}(\alpha<\lambda)$ be such that $T_{\alpha}$ is open and dense with respect to the dual Ellentuck topology (which is always possible by the Remark 1). For each $\alpha<\lambda$ let

$$
T_{\alpha}^{*}:=\left\{X \in(\omega)^{\omega}: \exists Y \in T_{\alpha}\left(X \sqsubseteq^{*} Y \wedge \neg(X \stackrel{*}{=} Y)\right)\right\} .
$$

It is easy to see, that for each $\alpha<\lambda$ the set $T_{\alpha}^{*}$ is open and dense with respect to the dual Ellentuck topology.

Let $U_{\alpha} \subseteq T_{\alpha}^{*}(\alpha<\lambda)$ be mao. Because $\lambda<\mathfrak{H}$, the set $\left\langle U_{\alpha}: \alpha<\lambda\right\rangle$ can not be shattering. Let for $\alpha<\lambda U_{\alpha}^{*}:=\left\{X \in(\omega)^{\omega}: \exists Z_{\alpha} \in U_{\alpha}\left(X \sqsubseteq^{*} Z_{\alpha}\right)\right\}$, then $U_{\alpha}^{*} \subseteq T_{\alpha}$ and $\bigcap_{\alpha<\lambda} U_{\alpha}^{*}$ is open and dense with respect to the dual Ellentuck topology:

$\bigcap U_{\alpha}^{*}$ is open: clear.

$\bigcap_{\alpha<\lambda}^{\alpha<\lambda} U_{\alpha}^{*}$ is dense: Let $(s, Z)^{\omega}$ be arbitrary. Because $\left\langle U_{\alpha}: \alpha<\lambda\right\rangle$ is not shattering, there is a $Y \in(s, Z)^{\omega}$ such that $\forall \alpha<\lambda \exists X_{\alpha} \in U_{\alpha}\left(Y \sqsubseteq^{*} X_{\alpha}\right)$. Hence, $Y \in$ $\bigcap_{\alpha<\lambda} U_{\alpha}^{*}$.

Further we have by construction

$$
\bigcap_{\alpha<\lambda} U_{\alpha}^{*} \cap \bigcup_{\alpha<\lambda} S_{\alpha}=\emptyset
$$

which completes the proof.

LEMMA $2.3 \mathfrak{H} \leq \mathbf{d}(\mathfrak{W})$

ProOF: Let $\left\langle T_{\alpha}: \alpha<\lambda<\mathfrak{H}\right\rangle$ be a sequence of open and dense sets with respect to the dual Ellentuck topology. Now the set $\bigcap_{\alpha<\lambda} U_{\alpha}^{*}$, constructed as in Lemma 2.2, is dense (and even open) and a subset of $\bigcap_{\alpha<\lambda} T_{\alpha}$. Therefore $\mathfrak{H} \leq \mathbf{d}(\mathfrak{W})$.

LEMMA $2.4 \operatorname{add}(\mathfrak{J}) \leq \mathfrak{H}$.

Proof: Let $\left\langle R_{\alpha}: \alpha<\mathfrak{H}\right\rangle$ be a shattering family and $P_{\alpha}:=\{X: \exists Y \in$ $\left.R_{\alpha}\left(X \sqsubseteq{ }^{*} Y\right)\right\}$.

For each $\alpha<\mathfrak{H}, P_{\alpha}$ is dense and open with respect to the dual Ellentuck topology:

$P_{\alpha}$ is open: clear.

$P_{\alpha}$ is dense: Let $(s, Z)^{\omega}$ be arbitrary and $X \in(s, Z)^{\omega}$. Because $R_{\alpha}$ is mao, there is a $Y \in R_{\alpha}$ such that $X^{\prime}:=X \sqcup Y \in(\omega)^{\omega}$. Now let $X^{\prime \prime} \stackrel{*}{=} X^{\prime}$ such that $X^{\prime \prime} \in(s, Z)^{\omega}$, then $X^{\prime \prime} \sqsubseteq^{*} Y$.

Now we show that $\bigcap_{\alpha<\mathfrak{H}} P_{\alpha}=\emptyset$ and therefore $\bigcup_{\alpha<\mathfrak{H}}\left((\omega)^{\omega} \backslash P_{\alpha}\right)=(\omega)^{\omega}$. Assume there is an $X \in \bigcap_{\alpha<\mathfrak{H}} P_{\alpha}$, then $\forall \alpha<\mathfrak{H} \exists \mathfrak{Y}_{\alpha} \in \mathfrak{R}_{\alpha}\left(\mathfrak{X} \sqsubseteq^{*} \mathfrak{Y}_{\alpha}\right)$. But this contradicts that $\left\langle R_{\alpha}: \alpha<\mathfrak{H}\right\rangle$ is shattering. 
LEMMA $2.5 \mathbf{d}(\mathfrak{W}) \leq \mathfrak{H}$.

Proof: In the proof of Lemma 2.4 we constructed a sequence $\left\langle P_{\alpha}: \alpha<\mathfrak{H}\right\rangle$ of open and dense sets with an empty intersection. Therefore $\bigcap_{\alpha<\mathfrak{H}} P_{\alpha}$ is not dense.

COROLLARY $2.6 \operatorname{cov}(\mathfrak{J}) \leq \mathfrak{H}$.

PROOF: In the proof of Lemma 2.4, in fact we proved that $\operatorname{cov}(\mathfrak{J}) \leq \mathfrak{H}$.

COROLlary $2.7 \operatorname{add}(\mathfrak{J})=\operatorname{cov}(\mathfrak{J})=\mathbf{d}(\mathfrak{W})=\mathfrak{H}$

ProOF: It is clear that $\operatorname{add}(\mathfrak{J}) \leq \operatorname{cov}(\mathfrak{J})$. By the Lemmas 2.3 and 2.5 we know that $\mathfrak{H}=\mathbf{d}(\mathfrak{W})$. Further by the Lemma 2.2 and the Corollary 2.6 it follows that $\mathfrak{H} \leq \operatorname{add}(\mathfrak{J}) \leq \operatorname{cov}(\mathfrak{J}) \leq \mathfrak{H}$. Hence we have $\operatorname{add}(\mathfrak{J})=\operatorname{cov}(\mathfrak{J})=\mathbf{d}(\mathfrak{W})=\mathfrak{H}$.

COROLLARY 2.8 The union of less than $\mathfrak{H}$ completely dual-Ramsey sets is dualRamsey, but the union of $\mathfrak{H}$ completely dual-Ramsey sets can be a set, which does not have the dual-Ramsey property.

ProOF: Follows from Remark 1 and Corollary 2.7.

\section{On the consistency of $\mathfrak{H}>\omega_{1}$}

First we give some facts concerning the dual-Mathias forcing.

The conditions of dual-Mathias forcing are pairs $\langle s, X\rangle$ such that $s \in(\mathbb{N})$, $X \in(\omega)^{\omega}$ and $s \sqsubseteq X$, stipulating $\langle s, X\rangle \leq\langle t, Y\rangle$ if and only if $(s, X)^{\omega} \subseteq(t, Y)^{\omega}$. It is not hard to see that similar to Mathias forcing, the dual-Mathias forcing can be decomposed as $\mathfrak{W} * \mathrm{P}_{\tilde{D}}$, where $\mathfrak{W}$ is defined as above and $\mathrm{P}_{\tilde{\mathfrak{D}}}$ denotes dual-Mathias forcing with conditions only with second coordinate in $\tilde{\mathfrak{D}}$, where $\tilde{\mathfrak{D}}$ is an $\mathfrak{W}$-generic object.

Further, because dual-Mathias forcing has pure decision (cf. CSS), it is proper and has the Laver property and therefore adds no Cohen reals.

If we make an $\omega_{2}$-iteration of dual-Mathias forcing with countable support, starting from a model in which the continuum hypothesis holds, we get a model in which the dual-shattering cardinal $\mathfrak{H}$ is equal to $\omega_{2}$.

Let $V$ be a model of $\mathrm{CH}$ and let $P_{\omega_{2}}:=\left\langle P_{\alpha}, \dot{Q}_{\beta}: \alpha \leq \omega_{2}, \beta<\omega_{2}\right\rangle$ be a countable support iteration of dual-Mathias forcing, i.e. $\forall \alpha<\omega_{2}: \Vdash_{P_{\alpha}}$ "Q $\dot{Q}_{\alpha}$ is dual-Mathias forcing".

In the sequel we will not distinguish between a member of $\mathfrak{W}$ and its representative. In the proof of the following theorem, a set $C \subseteq \omega_{2}$ is called $\omega_{1}$-club if $C$ is unbounded in $\omega_{2}$ and closed under increasing sequences of length $\omega_{1}$.

THEOREM 2.9 If $G$ is $P_{\omega_{2}}$-generic over $V$, where $V \models \mathrm{CH}$, then $V[G] \models \mathfrak{H}=$ $\omega_{2}$. 
Proof: In $V[G]$ let $\left\langle D_{\nu}: \nu<\omega_{1}\right\rangle$ be a family of open dense subsets of $\mathfrak{W}$. Because dual-Mathias forcing is proper and by a standard Löwenheim-Skolem argument, we find a $\omega_{1}$-club $C \subseteq \omega_{2}$ such that for each $\alpha \in C$ and every $\nu<\omega_{1}$ the set $D_{\nu} \cap V\left[G_{\alpha}\right]$ belongs to $V\left[G_{\alpha}\right]$ and is open dense in $\mathfrak{W}^{\mathfrak{V}\left[\mathfrak{G}_{\alpha}\right]}$. Let $A \in \mathfrak{W}^{\mathfrak{V}[\mathfrak{G}]}$ be arbitrary. By properness and genericity and because $P_{\omega_{2}}$ has countable support, we may assume that $A \in G(\alpha)^{\prime}$ for an $\alpha \in C$, where $G(\alpha)^{\prime}$ is the first component according to the decomposition of Mathias forcing of the $\dot{Q}_{\alpha}\left[G_{\alpha}\right]$-generic object determined by $G$. As $\alpha \in C, G(\alpha)^{\prime}$ clearly meets every $D_{\nu}\left(\nu<\omega_{1}\right)$. But now $X_{\alpha}$, the $\dot{Q}_{\alpha}$-generic partition (determined by $\left.G(\alpha)^{\prime \prime}\right)$ is below each member of $G(\alpha)^{\prime}$, hence below $A$ and in $\bigcap_{\nu<\omega_{1}} D_{\nu}$. Because $A$ was arbitrary, this proves that $\bigcap_{\nu<\omega_{1}} D_{\nu}$ is dense in $\mathfrak{W}$ and therefore $\mathbf{d}(\mathfrak{W})>\omega_{1}$. Again by properness of dual-Mathias forcing $V[G] \models 2^{\omega_{0}}=\omega_{2}$ and we finally have $V[G] \models \mathfrak{H}=\omega_{2}$.

In the model constructed in the proof of Theorem 2.9 we have $\mathfrak{H}>\mathfrak{t}$, where $\mathfrak{t}$ is the well-known tower number (for a definition of $\mathfrak{t} \mathrm{cf}$. $\mathrm{vDd}$ ). Moreover, we can show

COROLlaRY 2.10 The statement $\mathfrak{H}>\operatorname{cov}(\mathcal{M})$ is relatively consistent with ZFC, (where $\mathcal{M}$ denotes the ideal of meager sets).

ProOF: Because dual-Mathias forcing is proper and does not add Cohen reals, also forcing with $P_{\omega_{2}}$ does not add Cohen reals. Further it is known that $\mathfrak{t} \leq \operatorname{cov}(\mathcal{M})$ (cf. [PV] or [B]]). Now because forcing with $P_{\omega_{2}}$ does not add Cohen reals, in $V[G]$ the covering number $\operatorname{cov}(\mathcal{M})$ is still $\omega_{1}$ (because each real in $V[G]$ is in a meager set with code in $V$ ). This completes the proof.

REMARK 2: In $\mathrm{vDd}$ Theorem 3.1.(c) it is shown that $\omega \leq \kappa<\mathfrak{t}$ implies that $2^{\kappa}=2^{\omega_{0}}$. We do not have a similar result for the dual-shattering cardinal $\mathfrak{H}$. If we start our forcing construction $P_{\omega_{2}}$ with a model $V \models \mathrm{CH}+2^{\omega_{1}}=\omega_{3}$, then (again by properness of dual-Mathias forcing) $V[G] \models \mathfrak{H}=\omega_{2}=2^{\omega_{0}}<2^{\omega_{1}}=\omega_{3}$ (where $G$ is $P_{\omega_{2}}$-generic over $V$ ).

Remark: Recently Spinas showed in $[\mathrm{Sp}]$, that $\mathfrak{H}<\mathfrak{h}$ is consistent with ZFC. But it is still open if MA $+\neg \mathrm{CH}$ implies that $\omega_{1}<\mathfrak{H}$.

\section{On the dual-splitting cardinals $\mathfrak{S}$ and $\mathfrak{S}^{\prime}$}

Let $X_{1}, X_{2}$ be two partitions. We say $X_{1}$ splits $X_{2}$ if $X_{1} \| X_{2}$ and it exists a partition $Y \sqsubseteq X_{2}$, such that $X_{1} \perp Y$. A family $\mathcal{S} \subseteq(\omega)^{\omega}$ is called splitting if for each non-trivial $X \in(\omega)^{\omega}$ there exists an $S \in \mathcal{S}$ such that $S$ splits $X$. The dual-splitting cardinal $\mathfrak{S}$ (resp. $\mathfrak{S}^{\prime}$ ) is the least cardinal number $\kappa$, for which there exists a splitting family $\mathcal{S} \subseteq(\omega)^{\omega}$ (resp. $\mathcal{S} \subseteq(\omega)^{\underline{\omega}}$ ) of cardinality $\kappa$.

It is obvious that $\mathfrak{S} \leq \mathfrak{S}^{\prime}$.

First we compare the dual-splitting number $\mathfrak{S}^{\prime}$ with the well-known bounding number $\mathfrak{b}$ (a definition of $\mathfrak{b}$ can be found in $\mathrm{vD}]$ ).

TheOREM $3.1 \mathfrak{b} \leq \mathfrak{S}^{\prime}$. 
Proof: Assume there exists a family $\mathcal{S}=\left\{S_{\iota}: \iota<\kappa<\mathfrak{b}\right\} \subseteq(\omega) \underline{\omega}$ which is splitting. Let $B=\left\{b_{\iota}: \iota<\kappa\right\} \subseteq[\omega]^{\omega}$ a set of infinite subsets of $\omega$ such that $b_{\iota} \in S_{\iota}$ (for all $\iota<\kappa$ ). Let $f_{b_{\iota}} \in \omega^{\omega}$ be the (unique) increasing function such that range $\left(f_{b_{\iota}}\right)=b_{\iota}$. Because $\kappa<\mathfrak{b}$, the set $\left\{f_{b_{\iota}}: \iota<\kappa\right\}$ is not unbounded. Therefore there exists a function $d \in \omega^{\omega}$ such that $f_{b_{\iota}}<* d$ (for all $\iota<\kappa$ ). Now with the function $d$ we construct an infinite partition $D$. First we define an infinite set of pairwise disjoint finite sets $p_{i}(i \in \omega)$ :

$$
p_{i}:=\left[d^{i}(0), d^{i+1}\right)
$$

where $d^{i}$ denote the $i$-fold composition of $d$.

Now the blocks of $D$ are defined as follows:

$n$ is in the $k$ th block of $D$ iff $n \in p_{i} \wedge i-\max \left\{\frac{l}{2}(l+1)<i: l \in \omega\right\}=k$.

Because $d$ dominates $B$, for all $b_{\iota} \in B$ there exists a natural number $m_{\iota}$, such that for all $i>m_{\iota}$ : $d^{i}(0) \leq b_{\iota}\left(d^{i}(0)\right)<d^{i+1}(i)$ (cf. vDol p. 121). So, for all $i>m_{\iota}, p_{i} \cap b_{\iota} \neq \emptyset$ and therefore by the construction of the blocks of $D, b_{\iota}$ intersects each block of $D$. But this implies, that $D$ is not compatible with any element of $\mathcal{S}$ and so $\mathcal{S}$ can not be a splitting family.

COROLlary 3.2 It is consistent with ZFC, that $\mathfrak{s}<\mathfrak{S}^{\prime}$.

Proof: Because $\mathfrak{b} \leq \mathfrak{S}^{\prime}$ is provable in ZFC, it is enough to prove that $\mathfrak{s}<\mathfrak{b}$ is consistent with ZFC, which is proved in $\mathrm{Sh}$.

Now we show that $\operatorname{cov}(\mathcal{M}) \leq \mathfrak{S}$ (where $\mathcal{M}$ denotes the ideal of meager sets). In CMW it is shown that if $\kappa<\operatorname{cov}(\mathcal{M})$ and $\left\{X_{\alpha}: \alpha<\kappa\right\} \subseteq(\omega)^{\omega}$ is a family of partitions, then there exists $Y \in(\omega)^{\omega}$ such that $Y \perp X_{\alpha}$ for each $\alpha<\kappa$. This implies the following

Corollary $3.3 \operatorname{cov}(\mathcal{M}) \leq \mathfrak{S}$.

Proof: Let $S, Y \in(\omega)^{\omega}$. If $S \perp Y$, then $S$ does not split $Y$ and therefore a family of cardinality less than $\operatorname{cov}(\mathcal{M})$ can not be splitting.

As a corollary we get again a consistency result.

COROLlaRY 3.4 It is consistent with ZFC, that $\mathfrak{s}<\mathfrak{S}$.

Proof: If we make an $\omega_{1}$-iteration of Cohen forcing with finite support starting from a model $V \models \operatorname{cov}(\mathcal{M})=\omega_{2}=\mathfrak{c}$, we get a model in which $\omega_{1}=$ $\mathfrak{s}<\operatorname{cov}(\mathcal{M})=\omega_{2}=\mathfrak{c}$ holds. Hence, by Corollary 3.3, this is a model for $\omega_{1}=\mathfrak{s}<\mathfrak{S}=\omega_{2}$.

Until now we have $\operatorname{cov}(\mathcal{M}), \mathfrak{b} \leq \mathfrak{S}^{\prime}$, which would be trivial if one could show that $\mathfrak{S}^{\prime}=\mathfrak{c}$, where $\mathfrak{c}$ is the cardinality of $\mathcal{P}(\omega)$. But this is not the case (cf. CMW]). For the sake of completeness we will give now the notion of forcing used in CMW to construct a model in which we have $\mathfrak{S}^{\prime}<\mathfrak{c}$.

Let $\mathbf{Q}$ the notion of forcing defined as follows. The conditions of $\mathbf{Q}$ are pairs $\langle s, A\rangle$ such that $s \in(\mathbb{N}), A \in(\omega)^{<\omega}$ and $s \preceq A$, stipulating $\langle s, A\rangle \leq\langle t, B\rangle$ 
if and only if $t \preceq s$ and $B \sqsubseteq A$. ( $s$ is called the stem of the condition.) If $\left\langle s, A_{1}\right\rangle,\left\langle s, A_{2}\right\rangle$ are two $\mathbf{Q}$-conditions, then $\left\langle s, A_{1} \sqcup A_{2}\right\rangle \leq\left\langle s, A_{1}\right\rangle,\left\langle s, A_{2}\right\rangle$. Hence, two $\mathbf{Q}$-conditions with the same stem are compatible and because there are only countably many stems, the forcing notion $\mathbf{Q}$ is $\sigma$-centered.

Now we will see, that forcing with $\mathbf{Q}$ adds an infinite partition which is compatible with all old infinite partitions but is not contained in any old partition. (So, the forcing notion $\mathbf{Q}$ is in a sense like the dualization of Cohen forcing.)

LEMMA 3.5 If $G$ is $\mathbf{Q}$-generic over $V$, then $G \in(\omega)^{\underline{\omega}}$ and $V[G] \models \forall X \in(\omega)^{\omega} \cap$ $V\left(G \| X \wedge \neg\left(X \sqsubseteq^{*} G\right)\right)$.

Proof: Let $X \in V$ be an arbitrary, infinite partition. The set $D_{n}$ of $\mathbf{Q}$ conditions $\langle s, A\rangle$, such that

(i) at least one block of $s$ has more than $n$ elements,

(ii) at least $n$ blocks of $X$ are each the union of blocks of $A$,

(iii) there are at least $n$ different blocks $b_{i} \in X$, such that $\bigcup b_{i} \in s \sqcap X$,

is dense in $\mathbf{Q}$ for each $n \in \omega$. Therefore, at least one block of $G$ is infinite (because of (i)), $G$ is compatible with $X$ (because of (ii)) and $X$ is not coarser* than $G$ (because of (iii)). Now, because $X$ was arbitrary, the $\mathbf{Q}$-generic partition $G$ has the desired properties.

Because the forcing notion $\mathbf{Q}$ is $\sigma$-centered and each $\mathbf{Q}$-condition can be encoded by a real number, forcing with $\mathbf{Q}$ does neither collapse any cardinals nor change the cardinality of the continuum and we can prove the following

LEMMA 3.6 It is consistent with ZFC that $\mathfrak{S}^{\prime}<\mathfrak{c}$.

Proof: CMW If make an $\omega_{1}$-iteration of $\mathbf{Q}$ with finite support, starting from a model in which we have $\mathfrak{c}=\omega_{2}$, then the $\omega_{1}$ generic objects form a splitting family.

Even if a partition does not have a complement, for each non-trivial partition $X$ we can define a non-trivial partition $Y$, such that $X \perp Y$.

Let $X=\left\{b_{i}: i \in \omega\right\} \in(\omega)^{\omega}$ and assume that the blocks $b_{i}$ are ordered by their least element and that each block is ordered by the natural order. A block is called trivial, if it is a singleton. With respect to this ordering define for each non-trivial partition $X$ the partition $X^{<}$as follows.

If $X \in(\omega) \underline{\omega}$ then

$n$ is in the $i$ th block of $X^{<}$

iff

$n$ is the $i$ th element of a block of $X$,

otherwise 
$n, m$ are in the same block of $X^{<}$

iff

$n, m$ are both least elements of blocks of $X$.

It is not hard to see that for each non-trivial $X \in(\omega)^{\omega}, X \perp X^{<}$.

A family $\mathcal{W} \subseteq(\omega)^{\underline{\omega}}$ is called weak splitting, if for each partition $X \in(\omega)^{\omega}$, there is a $W \in \mathcal{W}$ such that $W$ splits $X$ or $W$ splits $X^{<}$. The cardinal number $w \mathfrak{S}$ is the least cardinal number $\kappa$, for which there exists a weak splitting family of cardinality $\kappa$. (It is obvious that $w \mathfrak{S} \leq \mathfrak{S}^{\prime}$.)

A family $U$ is called a $\pi$-base for a free ultra-filter $\mathcal{F}$ over $\omega$ provided for every $x \in \mathcal{F}$ there exists $u \in U$ such that $u \subseteq x$. Define

$\pi \mathfrak{U}:=\min \left\{|\mathfrak{U}|: \mathfrak{U} \subseteq[\omega]^{\omega}\right.$ is a $\pi$-base for a free ultra-filter over $\left.\omega\right\}$.

In BS it is proved, that $\pi \mathfrak{u}=\mathfrak{r}$ (see also Va for more results concerning $\mathfrak{r}$ ).

Now we can give an upper and a lower bound for the size of $w \mathfrak{S}$.

THEOREM $3.7 w \mathfrak{S} \leq \mathfrak{r}$.

Proof: We will show that $w \mathfrak{S} \leq \pi \mathfrak{u}$. Let $U:=\left\{u_{\iota} \in[\omega]^{\omega}: \iota<\pi \mathfrak{u}\right\}$ be a $\pi$-basis for a free ultra-filter $\mathcal{F}$ over $\omega$. W.l.o.g. we may assume, that all the $u_{\iota} \in U$ are co-infinite. Let $\mathcal{U}=\left\{Y_{u} \in(\omega)^{\omega}: u \in U \wedge Y_{u}=\left\{u_{i}: u_{i}=u \vee\left(u_{i}=\right.\right.\right.$ $\{n\} \wedge n \notin u)\}\}$. Now we take an arbitrary $X=\left\{b_{i}: i \in \omega\right\} \in(\omega)^{\omega}$ and define for every $u \in U$ the sets $I_{u}:=\left\{i: b_{i} \cap u \neq \emptyset\right\}$ and $J_{u}:=\left\{j: b_{j} \cap u=\emptyset\right\}$. It is clear that $I_{u} \cup J_{u}=\omega$ for every $u$.

If we find a $u \in U$ such that $\left|I_{u}\right|=\left|J_{u}\right|=\omega$, then $Y_{u}$ splits $X$. To see this, define the two infinite partitions

$$
Z_{1}:=\left\{a_{k}: a_{k}=\bigcup_{i \in I_{u}} b_{i} \vee \exists j \in J_{u} a_{k}=b_{j}\right\}
$$

and

$$
Z_{2}:=\left\{a_{k}: a_{k}=\bigcup_{j \in J_{u}} b_{j} \vee \exists i \in I_{u} a_{k}=b_{i}\right\}
$$

Now we have $X \sqcap Y_{u}=Z_{1}$ (therefore $Z_{1} \sqsubseteq X, Y_{u}$ ) and $Z_{2} \sqsubseteq X$ but $Z_{2} \perp Y_{u}$. (If each block of $b_{i}$ is finite, then we are always in this case.)

If we find an $x \in \mathcal{F}$ such that $\left|I_{x}\right|<\omega$ (and therefore $\left|J_{x}\right|=\omega$ ), then we find an $x^{\prime} \subseteq x$, such that $\left|I_{x}\right|=1$ and for this $i \in I_{x},\left|b_{i} \backslash x^{\prime}\right|=\omega$. (This is because $\mathcal{F}$ is a free ultra-filter.) Now take a $u \in U$ such that $u \subseteq x^{\prime}$ and we are in the former case for $X^{<}$. Therefore, $Y_{u}$ splits $X^{<}$.

If we find an $x \in \mathcal{F}$ such that $\left|J_{x}\right|<\omega$ (and therefore $\left|I_{x}\right|=\omega$ ), let $I(n)$ be an enumeration of $I_{x}$ and define $y:=x \cap \bigcup_{k \in \omega} b_{I(2 k)}$. Then $y \subseteq x$ and $|x \backslash y|=\omega$. Hence, either $y$ or $\omega \backslash y$ is a superset of some $u \in U$. But now $\left|J_{u}\right|=\omega$ and we are in a former case.

A lower bound for $w \mathfrak{S}$ is $\operatorname{cov}(\mathcal{M})$.

THEOREM $3.8 \operatorname{cov}(\mathcal{M}) \leq w \mathfrak{S}$. 
ProOF: Let $\kappa<\operatorname{cov}(\mathcal{M})$ and $\mathcal{W}=\left\{W_{\iota}: \iota<\kappa\right\} \subseteq(\omega) \underline{\omega}$. Assume for each $W_{\iota} \in \mathcal{W}$ the blocks are ordered by their least element and each block is ordered by the natural order. Further assume that $b_{i(\iota)}$ is the first block of $W_{\iota}$ which is infinite. Now for each $\iota<\kappa$ the set $D_{\iota}$ of functions $f \in \omega^{\omega}$ such that

$$
\begin{aligned}
\forall n, m, k \in \omega \quad & \exists h \in \omega t_{1} \in b_{n}, t_{2} \in b_{m}, t_{3}, t_{4} \in b_{h} \exists s \in b_{i(\iota)} \\
& f\left(t_{1}\right)=f\left(t_{3}\right) \wedge f\left(t_{2}\right)=f\left(t_{4}\right) \wedge\left|\left\{s^{\prime} \leq s: f\left(s^{\prime}\right)=f(s)\right\}\right|=k+1 .
\end{aligned}
$$

is the intersection of countably many open dense sets and therefore the complement of a meager set. Because $\kappa<\operatorname{cov}(\mathcal{M})$, we find an unbounded function $g \in \omega^{\omega}$ such that $g \in \bigcap_{\iota<\kappa} D_{\iota}$. The partition $G=\left\{g^{-1}(n): n \in \omega\right\} \in(\omega) \underline{\omega}$ is orthogonal with each member of $\mathcal{W}$ and for each $W_{\iota} \in \mathcal{W}$ and each $k \in \omega$, there exists an $s \in b_{i(\iota)}$, such that $s$ is the $k$ th element of a block of $G$. Hence, $\mathcal{W}$ can not be a weak splitting family.

\section{On the dual-reaping cardinals $\mathfrak{R}$ and $\mathfrak{R}^{\prime}$}

A family $\mathcal{R} \subseteq(\omega)^{\omega}$ is called reaping (resp. reaping'), if for each partition $X \in(\omega)^{\omega}$ (resp. $X \in(\omega) \underline{\omega}$ ) there exists a partition $R \in \mathcal{R}$ such that $R \perp X$ or $R \sqsubseteq^{*} X$. The dual-reaping cardinal $\mathfrak{R}$ (resp. $\mathfrak{R}^{\prime}$ ) is the least cardinal number $\kappa$, for which there exists a reaping (resp. reaping') family of cardinality $\kappa$.

It is clear that $\mathfrak{R}^{\prime} \leq \mathfrak{R}$. Further by finite modifications of the elements of a reaping family, we may replace $\sqsubseteq^{*}$ by $\sqsubseteq$ in the definition above.

If we cancel in the definition of the reaping number the expression " $R \sqsubseteq^{*} X$ ", we get the definition of an orthogonal family.

A family $\mathcal{O} \subseteq(\omega)^{\omega}$ is called orthogonal (resp. orthogonal'), if for each nontrivial partition $X \in(\omega)^{\omega}$ (resp. for each partition $X \in(\omega)^{\underline{\omega}}$ ) there exists a partition $O \in \mathcal{O}$ such that $O \perp X$. The dual-orthogonal cardinal $\mathfrak{O}$ (resp. $\mathfrak{O}^{\prime}$ ) is the least cardinal number $\kappa$, for which there exists a orthogonal (resp. orthogonal') family of cardinality $\kappa$. (It is obvious that $\mathfrak{O}^{\prime} \leq \mathfrak{O}$.) Note, that $\mathfrak{o}=\mathfrak{c}$, where $\mathfrak{c}$ is the cardinality of $\mathcal{P}(\omega)$ and $\mathfrak{o}$ is defined like $\mathfrak{O}$ but for infinite subsets of $\omega$ instead of infinite partitions. (Take the complements of a maximal antichain in $[\omega]^{\omega}$ of cardinality $\mathfrak{c}$. Because an orthogonal family must avoid all this complements, it has at least the cardinality of this maximal antichain.)

It is also clear that each orthogonal ${ }^{(\prime)}$ family is also a reaping ${ }^{(\prime)}$ family and therefore $\mathfrak{R}^{(\prime)} \leq \mathfrak{O}^{(\prime)}$. Further one can show that $\mathfrak{R}^{\prime}$ is uncountable (cf. CMW]). Now we show that $\mathfrak{O}^{\prime} \leq \mathfrak{d}$, where $\mathfrak{d}$ is the well-known dominating number (for a definition cf. $\mathrm{vDd}$ ), and that $\operatorname{cov}(\mathcal{M}) \leq \mathfrak{O}^{\prime}$.

LEMMA $4.1 \mathfrak{O}^{\prime} \leq \mathfrak{d}$.

Proof: Let $\left\{d_{\iota} \in \omega^{\omega}: \iota<\mathfrak{d}\right\}$ be a dominating family. Then it is not hard to see that the family $\left\{D_{\iota}: \iota<\kappa\right\} \subseteq(\omega)^{\omega}$, where each $D_{\iota}$ is constructed from $d_{\iota}$ like $D$ from $d$ in the proof of Theorem 3.1, is an orthogonal family.

Let $\mathfrak{i}$ be the least cardinality of an independent family (a definition and some results can be found in $\mathrm{Ku}$ ), then 
LEMMA $4.2 \mathfrak{O} \leq \mathfrak{i}$.

Proof: Let $I \subseteq[\omega]^{\omega}$ be an independent family of cardinality i. Let $I^{\prime}:=\{r \in$ $\left.[\omega]^{\omega}: r \stackrel{*}{=} \bigcap \mathcal{A} \backslash \bigcup \mathcal{B}\right\}$, where $\mathcal{A}, \mathcal{B} \in[I]^{<\omega}, \mathcal{A} \neq \emptyset, \mathcal{A} \cap \mathcal{B}=\emptyset$ and $r \stackrel{*}{=} x$ means $|(r \backslash x) \cup(x \backslash r)|<\omega$. It is not hard to see that $\left|I^{\prime}\right|=|I|=\mathfrak{i}$. Now let $\mathcal{I}=\mathcal{I}_{1} \cup \mathcal{I}_{2}$ where $\mathcal{I}_{1}:=\left\{X_{r} \in(\omega)^{\omega}: r \in I^{\prime} \wedge X_{r}=\left\{b_{i}: b_{i}=r \vee\left(b_{i}=\{n\} \wedge n \notin r\right)\right\}\right\}$ and $\mathcal{I}_{2}:=\left\{Y_{r}: \exists X_{r} \in \mathcal{I}_{1}\left(Y_{r}=X_{r}^{\angle}\right)\right\}$. We see, that $\mathcal{I} \subseteq(\omega)^{\omega}$ and $|\mathcal{I}|=\mathfrak{i}$. It leave to show that $\mathcal{I}$ is an orthogonal family.

Let $Z \in(\omega)^{\omega}$ be arbitrary and let $r:=\operatorname{Min}(Z)$. If $r \in I^{\prime}$, then $X_{r} \perp Z$ (where $\left.X_{r} \in \mathcal{I}_{1}\right)$. And if $r \notin I^{\prime}$, then there exists an $r^{\prime} \in I^{\prime}$ such that $r \cap r^{\prime}=\emptyset$. But then $Y_{r^{\prime}} \perp Z$ (where $Y_{r^{\prime}} \in \mathcal{I}_{2}$ ).

Because $\mathfrak{R} \leq \mathfrak{O}$, the cardinal number $\mathfrak{i}$ is also an upper bound for $\mathfrak{R}$. But for $\mathfrak{R}$, we also find another upper bound.

LEMMA $4.3 \mathfrak{R} \leq \mathfrak{r}$.

Proof: Like in Theorem 3.7 we show that $\mathfrak{R} \leq \pi \mathfrak{u}$. Let $U:=\left\{u_{\iota} \in[\omega]^{\omega}: \iota<\right.$ $\pi \mathfrak{u}\}$ be as in the proof of Theorem 3.7 and let $\mathcal{U}=\left\{Y_{u} \in(\omega)^{\omega}: u \in U \wedge Y_{u}=\right.$ $\left.\left\{u_{i}: u_{i}=\omega \backslash u \vee\left(u_{i}=\{n\} \wedge n \in u\right)\right\}\right\}$. Take an arbitrary partition $X \in(\omega)^{\omega}$. Let $r:=\operatorname{Min}(X)$ and $r_{1}:=\{n \in r:\{n\} \in X\}$. If we find a $u \in U$ such that $u \subseteq r_{1}$, then $Y_{u} \sqsubseteq X$. Otherwise, we find a $u \in U$ such that either $u \subseteq \omega \backslash r$ or $u \subseteq r \backslash r_{1}$ and in both cases $Y_{u} \perp X$.

Now we will show, that it is consistent with ZFC that $\mathfrak{O}$ can be small. For this we first show, that a Cohen real encode an infinite partition which is orthogonal to each old non-trivial infinite partition. (This result is in fact a corollary of Lemma 5 of CMW.)

LEMMA 4.4 If $c \in \omega^{\omega}$ is a Cohen real over $V$, then $C:=\left\{c^{-1}(n): n \in \omega\right\} \in$ $(\omega) \underline{\omega} \cap V[c]$ and $\forall X \in(\omega)^{\omega} \cap V(\neg(X \stackrel{*}{=}\{\omega\}) \rightarrow C \perp X)$.

Proof: We will consider the Cohen-conditions as finite sequences of natural numbers, $s=\{s(i): i<n<\omega\}$. Let $X=\left\{b_{i}: i \in \omega\right\} \in V$ be an arbitrary, non-trivial infinite partition. The set $D_{n, m}$ of Cohen-conditions $s$, such that

(i) $|\{i: s(i)=0\}| \geq n$,

(ii) $\exists k>n \exists i(s(i)=k)$,

(iii) $\exists a_{n} \in b_{n} \exists a_{m} \in b_{m} \exists l \exists a_{1}, a_{2} \in b_{l}\left(s\left(a_{n}\right)=s\left(a_{1}\right) \wedge s\left(a_{m}\right)=s\left(a_{2}\right)\right)$,

is a dense set for each $n, m \in \omega$. Now, because $X$ was arbitrary, the infinite partition $C$ is orthogonal to each infinite partition which is in $V$. (Note that because of (i), $C \in(\omega) \underline{\omega}$.)

Now we can show, that $\mathfrak{O}$ can be small.

LEMMA 4.5 It is consistent with ZFC that $\mathfrak{O}<\operatorname{cov}(\mathcal{M})$. 
PROOF: If make an $\omega_{1}$-iteration of Cohen forcing with finite support, starting from a model in which we have $\mathfrak{c}=\omega_{2}=\operatorname{cov}(\mathcal{M})$, then the $\omega_{1}$ generic objects form an orthogonal family. Now because this $\omega_{1}$-iteration of Cohen forcing does not change the cardinality of $\operatorname{cov}(\mathcal{M})$, we have a model in $\omega_{1}=\mathfrak{O}<\operatorname{cov}(\mathcal{M})=$ $\omega_{2}$ holds.

Because $\mathfrak{R} \leq \mathfrak{O}$ we also get the relative consistency of $\mathfrak{R}<\operatorname{cov}(\mathcal{M})$. Note that this is not true for $\mathfrak{r}$.

As a lower bound for $\mathfrak{R}^{\prime}$ we find $\mathfrak{p}$, where $\mathfrak{p}$ is the pseudo-intersection number (a definition of $\mathfrak{p}$ can be found in $[\mathrm{vDd}$ ).

LEMMA $4.6 \mathfrak{p} \leq \mathfrak{R}^{\prime}$.

ProOF: In $\mathrm{Be}]$ it is proved that $\mathfrak{p}=\mathfrak{m}_{\sigma \text {-centered }}$, where

$$
\mathfrak{m}_{\sigma \text {-centered }}=\min \{\kappa: \text { :MA }(\kappa) \text { for } \sigma \text {-centered posets" fails }\} .
$$

Let $\mathcal{R}=\left\{R_{\iota}: \iota<\kappa<\mathfrak{p}\right\}$ be a set of infinite partitions. Now remember that the forcing notion $\mathbf{Q}$ (defined in section 3 ) is $\sigma$-centered and because $\kappa<\mathfrak{p}$ we find an $X \in(\omega)^{\underline{\omega}}$ such that $\mathcal{R}$ does not reap $X$.

As a corollary we get

COROLlary 4.7 If we assume MA, then $\mathfrak{R}^{\prime}=\mathfrak{c}$.

Proof: If we assume MA, then $\mathfrak{p}=\mathfrak{c}$.

\section{What's about towers and maximal (almost) or- thogonal families?}

Let $\kappa_{\text {mao }}$ be the least cardinal number $\kappa$, for which there exists an infinite mao family of cardinality $\kappa$. And let $\kappa_{\text {tower }}$ be the least cardinal number $\kappa$, for which there exists a family $\mathcal{F} \subseteq(\omega)^{\omega}$ of cardinality $\kappa$, such that $\mathcal{F}$ is well-ordered by $\sqsubseteq^{*}$ and $\neg \exists Y \in(\omega)^{\omega} \forall X \in \mathcal{F}\left(Y \sqsubseteq^{*} X\right)$.

Now Krawczyk proved that $\kappa_{\text {mao }}=\mathfrak{c}(\mathrm{cf}$. CMW $)$ and Carlson proved that $\kappa_{\text {tower }}=\omega_{1}$ (cf. [Ma]). So, these cardinals do not look interesting. But what happens if we cancel the word "almost" in the definition of $\kappa_{m a o}$ ?

A family $\mathcal{F} \subseteq(\omega)^{\omega}$ (resp. $\left.\mathcal{F} \subseteq(\omega)^{\underline{\omega}}\right)$ is a maximal anti-chain in $(\omega)^{\omega}$ (resp. $(\omega) \underline{\omega})$, if $\mathcal{F}$ is a maximal infinite family of pairwise orthogonal partitions. Let $\kappa_{A}$ (resp. $\kappa_{A^{\prime}}$ ) be the least cardinality of a maximal anti-chain in $(\omega)^{\omega}$ (resp. $(\omega) \underline{\omega})$.

Note that the corresponding cardinal for infinite subsets of $\omega$ would be equal to $\omega$.

First we know that $\operatorname{cov}(\mathcal{M}) \leq \kappa_{A}, \kappa_{A^{\prime}}$ (which is proved in CMW]) and $\mathfrak{b} \leq \kappa_{\mathfrak{A}^{\prime}}$ (which one can prove like Theorem 3.1). Further it is not hard to see that $\kappa_{A} \leq \kappa_{A^{\prime}}$.

But these results concerning $\kappa_{A}$ and $\kappa_{A^{\prime}}$ are also not interesting, because Spinas showed in Sp that $\kappa_{A}=\kappa_{A^{\prime}}=\mathfrak{c}$. 


\section{The diagram of the results}

Now we summarize the results proved in this article together with other known results.

splitting:

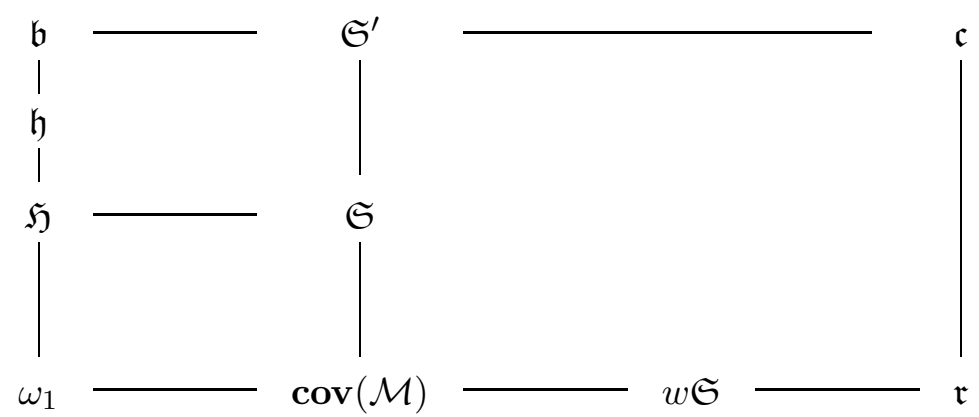

reaping:

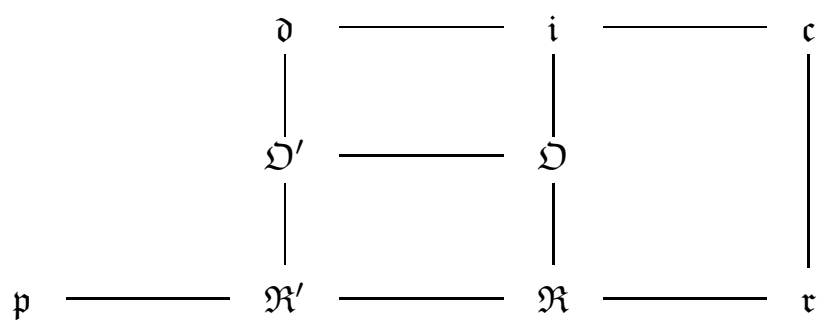

(In the diagrams, the invariants grow larger, as one moves up or to the right.)

\section{Consistency results:}

- $\operatorname{cov}(\mathcal{M})<\mathfrak{H} ; \mathfrak{H}<\mathfrak{h} ; \mathfrak{H}<\operatorname{cov}(\mathcal{M})$ (this is because $\mathfrak{h}<\operatorname{cov}(\mathcal{M})$ is consistent with ZFC)

- $\mathfrak{s}<\mathfrak{S} ; \mathfrak{S}^{\prime}<\mathfrak{c}$

- $\mathfrak{O}<\operatorname{cov}(\mathcal{M})$

Note ADded In PRoOf: Recently, Jörg Brendle informed me that he has proved, that $\mathbf{M A}+\mathfrak{H}<2^{\aleph_{0}}$ is consistent with ZFC.

\section{References}

[BPS] B. Balcar, J. Pelant and P. Simon: The space of ultrafilters on $N$ covered by nowhere dense sets. Fund. Math. 110(1980), 11-24.

[BS] B. BALCAR AND P. Simon: On minimal $\pi$-character of points in extremally disconnected compact spaces. Topology and its Applications 41(1991), 133-145.

[BJ] T. Bartoszyński and H. Judah: "Set Theory: the structure of the real line." A. K. Peters, Wellesley 1995. 
[Be] M. G. Bell: On the combinatorial principle $P(\mathfrak{c})$. Fund. Math. 114(1981), 149-157.

[CS] T. J. Carlson and S. G. Simpson: A Dual Form of Ramsey's Theorem. Adv. in Math. 53(1984), 265-290.

[CMW] J. Cichon, B. Majcher and B. Weglorz: Dualizations of van Douwen diagram, (preprint).

[Je1] T. Jech: "Multiple Forcing." Cambridge University Press, Cambridge 1987.

[Je2] T. Jech: "Set Theory." Academic Press, London 1978.

[Ku] K. Kunen: "Set Theory, an Introduction to Independence Proofs." North Holland, Amsterdam 1983.

[Ma] P. Matet: Partitions and Filters. J. Symbolic Logic 51(1986), 12-21.

[PV] Z. Piotrowski and A. Szymański: Some remarks on category in topological spaces. Proc. Amer. Math. Soc. 101(1987), 156-160.

[Pl] S. PlewiK: On completely Ramsey sets. Fund. Math. 127(1986), 127132.

[Sh] S. Shelah: On cardinal invariants of the continuum Cont. Math. 31(1984), 183-207.

[Sp] O. SpINAs: Partition numbers, (preprint).

[vDo] E. K. van Douwen: The integers and topology, in "Handbook of settheoretic topology," (K. Kunen and J. E. Vaughan, Ed.), pp. 111-167, North-Holland, Amsterdam 1990.

[Va] J. E. Vaughan: Small uncountable cardinals and topology, in "Open problems in topology," (J.van Mill and G. Reed, Ed.), pp. 195-218, North-Holland, Amsterdam 1990.

Lorenz Halbeisen

Departement Mathematik

ETH-Zentrum

8092 Zürich

Switzerland

E-mail: halbeis@math.ethz.ch 